\title{
¿Cómo comprometer el potencial del estudiante? \\ Proporcionar aprendizaje a partir de las diferencias
}

\section{Mariana Ramírez Sánchez}

Centro de Desarrollo Humano (CEDHU)

enjoythat25@gmail.com

Received: February 27, 2021

Accepted: May 4, 2021

\section{How to cite this article (APA, 7th ed.):}

Ramírez, M. (2021). ¿Cómo comprometer el potencial del estudiante? Proporcionar aprendizaje a partir de las diferencias. ENLETAWA Journal, 14(1), 126-132.

En el día a día, mi quehacer como docente, viene acompañada de trabajo dispendioso dentro de una larga cantidad de estudiantes, entre 30 a 35. Al ser grupos heterogéneos, uno de los problemas presentados en la relación docente-estudiante suele centrarse en proporcionar aprendizaje para todos y proporcionar oportunidades para que cada estudiante progrese individualmente; teniendo en cuenta sus edades, expectativas, necesidades, debilidades, rango de habilidades cognitivas, estilos de aprendizaje, intereses, actitudes, opiniones, sentimientos y comportamientos. En mi experiencia como docente de español como lengua materna e inglés como lengua extranjera, he evidenciado el funcionamiento y desarrollo de algunas alternativas pedagógicas dentro del aula de clase para comprometer el potencial de cada estudiante. Entre ellas, mantener vivo el interés de los estudiantes por medio de la variación, adaptación de actividades y ejercicios de acuerdo con los niveles de velocidad de trabajo en cada uno, el enfoque dialógico-crítico (y la formulación de preguntas abiertas), la importancia de exigir para ascender, realimentar más no corregir y analizar continuamente la propia identidad como ser humano y profesional. 
Primeramente, la variación dentro del salón de clases es sin duda alguna una herramienta muy importante para proporcionar aprendizaje. Como se menciona anteriormente, un salón numeroso de 30 a 35 estudiantes es heterogéneo por el simple hecho que cada estudiante es diferente. Pero... ¿Qué significa variar? ¿Cómo asegurarse de que todos los estudiantes aprenden cuando todos son tan diferentes? ¿Cómo asegurarse de proporcionar oportunidades para que cada estudiante progrese por su cuenta? Y ¿Cómo encontrar los materiales adecuados? Pude observar, que, en ciertos casos, algunos estudiantes son más rápidos que otros y comienzan a hablar con sus vecinos cuando terminan una tarea $o$ ejercicio dado. Algo demasiado difícil, es aburrido para ellos; y algo demasiado fácil, también. En realidad, es un verdadero desafío mantener a un grupo de estudiantes interesados en aprender, y a la vez, crear en ellos una expresión de curiosidad; ya que no existen verdades ni soluciones absolutas en el campo educativo. Ur (1999) afirma que: 'Una lección variada, además de ser más interesante y placentera tanto para los maestros como para los estudiantes, también abarca una gama más amplia de estilos y estrategias de aprendizaje que pueden proporcionar cambios refrescantes en el tipo de actividad mental y física exigida" (p. 216). Teniendo esta afirmación en cuenta, opté por planear lecciones con variedad en los temas, las actividades, la organización del aula y el tipo de materiales diseñados. Variación en los temas, porque un tema que puede ser interesante para un grupo de estudiantes, para otros no lo es. Variación en las actividades (a veces sencillas, otras veces más retadoras, pero no siempre en el mismo nivel). Variación en la organización de actividades, unas veces grupales y otras individuales ya que a algunos estudiantes les gusta trabajar por su cuenta y a otros les gusta trabajar en equipo. Variación en el tipo de materiales (algunas veces usar material electrónico, otras veces lápiz y papel, juegos, la pizarra, libros, música, etc.). Los materiales visuales son vitales porque los estudiantes siempre tendrán algo que observar, los ojos son un canal de aprendizaje muy poderoso. La variación crea interés en los estudiantes, ya que saben que cada clase habrá algo nuevo por hacer y descubrir. Gracias a la variación, los estudiantes participan activamente en cada clase y se evidencia que sus preferencias siempre serán diferentes; algo que funciona hoy, quizás mañana no.

En segundo lugar, para proporcionar aprendizaje a todos los estudiantes es muy 
importante adoptar actividades y ejercicios de acuerdo con los niveles de velocidad/ritmo de trabajo en cada uno. Según Ur (1999) "[hay] dos estrategias que permiten a los estudiantes aprender a su propio ritmo y nivel incluso dentro de un ejercicio convencional dirigido por el maestro. El primero es «comenzar donde quieran» y el segundo es «establecer el tiempo, no la cantidad»" (p. 221). Para la primera estrategia, en algunas de las actividades planteadas dentro del aula, en lugar de preguntar: ¿Quién ya desarrollo el taller? o ¿Quién pudo desarrollar la pregunta 1 ? se les puede dar a los estudiantes un par de minutos para leer todas las preguntas de un taller o ejercicio, y si quieren responder alguna de las preguntas, no solo la 1, pueden levantar la mano. Así, los estudiantes menos avanzados o quienes normalmente tienen miedo al hablar, pueden ir directamente a las preguntas más fáciles o a las preguntas con las cuales ellos se sientan más cómodos para responder. Cuando se realiza este ejercicio, los estudiantes se sienten importantes y confían en ellos mismos. Para la segunda estrategia, logré evidenciar que dar un límite de tiempo es mucho mejor que dar una cantidad de trabajo límite. En lugar de plantear: "Para la tarea, desarrollen los ejercicios 1,2 y 3 de la página 26 " se puede plantear: "Para la tarea, desarrollen todo lo que puedan de los ejercicios de la página 26 en 30 minutos." Esto implica que de vez en cuando se debe confiar en que los estudiantes tendrán un buen desempeño de acuerdo con las instrucciones que el maestro brinde. Los estudiantes de trabajo más lento, harán menos. Los de trabajo más rápido, harán más; pero todos trabajarán de acuerdo con su propio ritmo y capacidad. Si al revisar la tarea ellos hicieron 3 ejercicios, está bien. Si hicieron 5 o más, está bien. Esta estrategia la encontré mucho más fructífera para los estudiantes, ya que muestran gran compromiso para desarrollar los trabajos y tareas propuestos en clase de acuerdo con su propio ritmo. De lo contrario, si se les da una cantidad de trabajo y ellos no logran terminar, podrían sentirse frustrados.

En tercer lugar, los estudiantes pueden progresar individual $\mathrm{y}$ colectivamente gracias a el enfoque dialógico-crítico y la formulación de preguntas abiertas a partir del análisis de un texto. En palabras de Gonzáles (2018) es posible afirmar que:

El enfoque pedagógico dialógicocrítico, se consolida a través de todas las fases del proceso didáctico, pues [...] se muestra permanente interacción comunicativa entre el docente y sus estudiantes, a quienes se 
les exhorta a participar, a proponer, a producir textos, a socializar $\mathrm{y}$, especialmente, a expresar libremente sus posturas frente a los textos leídos y al mismo contenido temático de la clase. Dicho enfoque se apoya en el método inductivo. Por cuanto, a partir de observaciones de la lectura de textos $\mathrm{y}$, fundamentalmente, de los interrogantes formulados por el maestro, los estudiantes piensan, recuerdan, asocian, y así logran configurar y expresar sus propias ideas, sin esperar que siempre sea el docente quien las exponga, o transmita. (p. 129)

En la misma línea, formular preguntas abiertas a partir de la lectura, da espacio para proporcionar varias respuestas a una sola pregunta. Las preguntas cerradas no pueden generar respuestas diferentes porque solo hay una respuesta correcta (Ur, 1999). Formulando preguntas abiertas, el enfoque dialógico-crítico es desarrollado, ya que los estudiantes responden preguntas en diferentes niveles, además de expresar sus propias experiencias, preferencias, opiniones e ideas respecto a un tema en específico a través de la lectura; y esto responde a un proceso de interacción para el desarrollo del acto comunicativo (Avendaño, 2012).
Asimismo, los demás estudiantes escuchan y aprenden de las demás perspectivas que sus compañeros tienen y esto se transforma en un aprendizaje colaborativo en el que los estudiantes trabajaban juntos para crear un consenso.

En cuarto lugar, exigir a los estudiantes para ascender es una tarea fundamental para proporcionar aprendizaje y que los estudiantes avancen por su cuenta. Exigir se trata de usar cualquier actividad o trabajo en el aula para desafiar a cada estudiante, individualmente, a mejorar. Es decir, "exigir para ascender es empujar cuidadosamente a los estudiantes hacia adelante desde el punto en el que se encuentran" (Scrivener, 2012, p. 51). Los estudiantes tienen grados masivos de potencial, interés y capacidad de aprendizaje. Constantemente, me preocupo por analizar si como docente estoy comprometiendo todo el potencial de aprendizaje que los estudiantes tienen. Eventualmente, pienso constantemente en el hecho de "empujar cuidadosamente" a los estudiantes y hacer ajustes en lo que ellos creen que hicieron bien, pero que en realidad pueden mejorar. De esta manera, el aprendizaje avanza, ya que este no es fijo y está cambiando todo el tiempo. Cabe aclarar, que exigir no es un método, es una idea y el objetivo es ascender. 
Con pequeños “empujones" los estudiantes pueden ser ligeramente mejores. Como docentes, algunas veces sabemos cómo proporcionar tareas o actividades, pero no siempre tenemos claro dónde está el aprendizaje. Algunas veces, verificamos las respuestas de los estudiantes, pero no el proceso de aprendizaje que tuvieron para responder. Es necesario encontrar los lugares o áreas en que los estudiantes son débiles para empujarlos cuidadosamente a ser mejores.

En quinto lugar, he comprobado que es importante romper con el esquema "correcto/incorrecto" ya que este es un concepto que personalmente, considero como limitado para proporcionar aprendizaje. El docente, en lugar de obsesionarse con la búsqueda de respuestas correctas, puede encontrar formas de ayudar a los estudiantes individualmente a avanzar desde donde sea que estén, por eso la realimentación es una mejor opción en lugar de corregir (Scrivener, 2012). Al decir que algo es correcto o incorrecto, habría que analizar la cantidad de pasos que existen entre saber y no saber nada en definitivo. El estudiante débil no es el que siempre necesita un refuerzo y el excelente no es el que siempre levanta la mano o el que termina una tarea primero. Con cualquier cosa que diga o haga un estudiante es necesario "empujarlo" cuidadosamente para que obtenga un mejor resultado. En este sentido, los estudiantes no deberían recibir realimentación con un: eso es "correcto" o “incorrecto", podrían recibir realimentación para ser ligeramente mejores desde lo que tienen, desde lo que logran hacer o decir por su cuenta. Por consiguiente, si se realimenta a los estudiantes, no se hieren susceptibilidades, los estudiantes no se frustran $y$ no se sienten incapaces de ascender. Ahora, ascender, se centra en una mejoría de todo lo que el estudiante hace, exista un error o no.

El estudiante no necesita cometer un error para tener la necesidad de mejorar, por eso la realimentación es más poderosa que un elogio porque cuando un maestro elogia a un estudiante cuando este obtiene un buen primer resultado, este no tendría ninguna razón para mejorar. (Scrievener, 2012, p. 54)

Un claro ejemplo es mantener una pregunta abierta, escuchar a los estudiantes, y según lo que digan, no siempre es bueno validar con un "es correcto". Cuando se pone un sello de validación o se elogia la respuesta de un estudiante, la pregunta se extingue, no hay a donde ir y no tiene sentido pedirle a otro estudiante una opinión o respuesta alternativa. Con esto, podría decirse que el 
elogio es adecuado siempre y cuando el estudiante haya tenido un proceso de trabajo en la mejora de sus producciones. Siempre se puede mejorar, aún cuando los estudiantes crean que lo que hicieron está perfecto. En suma, la realimentación es diferente a la corrección, ya que la corrección transforma algo que está mal en algo correcto y la realimentación ayuda a mejorar desde lo que cada estudiante logra hacer.

En último lugar, analizar mi identidad como ser humano y profesional en el campo educativo, es una de las herramientas más útiles para saber de qué manera proporcionar aprendizaje y cómo mejorar en el proceso. Como docente siempre estoy en un proceso de identificar problemas, reflexionando, planificando y actuando sobre ellos. Para mí, no hay una fórmula secreta para enseñar, para controlar a los estudiantes, ni para hacer que se concentren y disfruten todas las clases. A pesar de esto, la labor del docente no termina en una clase, la labor del docente es constante, ya que siempre está en continuo aprendizaje. Como docente, no dejo de estudiar, no dejo de leer profunda y constantemente, no dejo de buscar información, no dejo de compartir experiencias con colegas, no dejo de innovar, siempre creo una lista de cosas que quiero explorar y hacer, procuro ser organizada y hago de mi profesión una prioridad.

En resumen, proporcionar aprendizaje y proporcionar oportunidades para que cada estudiante progrese por su cuenta sin duda alguna es una tarea ardua. Gracias a la diversidad que pude encontrar en mis salones de clase, comprendí que como docente puedo enseñar a mis estudiantes diferentes formas de entender y ver el mundo a través de una gran cantidad de perspectivas. Mis estudiantes actúan críticamente cuando se enfrentan a varias preguntas sobre un tema o problema en específico en el cual deben opinar y en el cual tienen el derecho a expresarse libremente. Proporcionar aprendizaje en equidad hace que mi identidad como docente se centre en educar seres humanos para la vida; siempre destacando sus fortalezas por encima de sus debilidades. 


\section{ABOUT THE AUTHOR}

Mariana Ramírez Sánchez holds a B.A. in Modern Languages teaching from the Universidad Pedagógica y Tecnológica de Colombia. She is an active member of Knowledge in Action- KIA research group. She is interested in exploring ways of designing and applying contextualized materials under the scope of Social Justice and antiracist education in order to develop more conscious and humanistic pedagogical practices in the EFL classroom.

\section{Referencias}

Avendaño, G. S. (2012). La lectura y la escritura genuina: Un modelo teórico-práctico para su dinamización y cualificación. Ponencia presentada en el $V$ Congreso Internacional de Letras, Transformaciones Culturales. Debates de la teoría, la crítica y la lingüística. Universidad de Buenos Aires -UBA-.
Gonzáles, O. (2018). Convergencia de enfoques lingüístico, psicolingüístico y sociocultural en la lectura crítica: Una guía didáctica para el docente. [Tesis de Maestría, Universidad Pedagógica y Tecnológica de Colombia].

Scrivener, J. (2012). Demand-high teaching. The European Journal of Applied Linguistics and TELF, 47-57.

Ur, P. (1999). Large heterogeneous classes: A course in language teaching trainee book (Cambridge Teacher Training and Development). Cambridge University Press. 